\title{
Patient-controlled epidural analgesia versus conventional epidural analgesia after total hip replacement - a randomized trial
}

\author{
Jan Maca ${ }^{a}$, Jan Neiser ${ }^{a}$, Lenka Grasslovac, Michaela Trlicovad , Dana Streitova ${ }^{\mathrm{a}, \mathrm{b}}$, Renata Zoubkova ${ }^{\mathrm{a}, \mathrm{b}}$
}

\begin{abstract}
Aims. Patient-controlled analgesia (PCA) is usually considered a better option for pain management compared to conventional analgesia. The beneficial effect of PCA has been assessed in a number of studies; however, the results are inconsistent. The goal of this study was to compare of patient-controlled epidural analgesia (PCEA) to conventional epidural analgesia after total hip replacement (THR).

Methods. This prospective study was performed at the Department of Anesthesia and Intensive Care Medicine at a tertiary university hospital. After THR, patients were admitted to the intensive care unit (ICU) and randomized to one of two groups (PCEA and non-PCEA). Postoperative pain in the PCEA group was treated using a standardized protocol, while the analgesia in the non-PCEA group was based on physician prescription according to the patient's clinical condition. The total consumption of analgesics, patients' satisfaction, pain intensity, and analgesia-related complications were recorded for $24 \mathrm{~h}$ after surgery.

Results. The final sample consisted of 111 patients (PCEA group, $n=55$ and non-PCEA group, $n=56$ ). The PCEA group had significantly lower total consumption of analgesic mixtures $(0.9 \pm 0.3$ and $1.3 \pm 0.4 \mathrm{~mL} / \mathrm{kg}$ per day, $P<0.001)$.There was greater patient satisfaction $(P<0.001)$ in the PCEA group. The mean pain intensity over 24 hours postoperatively was similar for both groups $(P=0.14)$. There was no significant difference in rate of analgesia-related complications between the groups (hypotension, $P=0.14$; bradypnea, $P=0.11$ ).

Conclusion. Compared to conventional epidural analgesia based on physician prescription, PCEA led to less total analgesic consumption and greater patient satisfaction after THR.
\end{abstract}

Key words: patient-controlled analgesia, epidural analgesia, analgesics, drug consumption, total hip replacement, pain relief

Received: August 7, 2018; Accepted: October 19, 2018; Available online: November 6, 2018

https://doi.org/10.5507/bp.2018.068

(c) 2020 The Authors; https://creativecommons.org/licenses/by/4.0/

${ }^{a}$ Department of Anesthesiology and Intensive Care Medicine, University Hospital of Ostrava, Czech Republic

${ }^{b}$ Department of Nursing, St. Elisabeth University of Health and Social Sciences, Bratislava, Slovak Republic

'Department of Rehabilitation and Physical Therapy, University Hospital of Ostrava, Czech Republic

${ }^{d}$ Faculty of Medicine, University of Ostrava, Czech Republic

Corresponding author: Jan Maca, e-mail: jan.maca@fno.cz

\section{INTRODUCTION}

Total hip replacement (THR) is usually followed by significant postoperative pain. Postoperative analgesia is therefore a fundamental part of postoperative management after THR, and may affect postoperative outcomes. Poorly managed postoperative pain increases the risk of developing chronic pain, postoperative morbidity, complications, costs, and length of hospital stay ${ }^{1,2}$. In contrast, adequate analgesia has a positive effect on patient comfort, including early mobilization ${ }^{3}$. With respect to the mode of analgesia, intravenous (i.v.) patient-controlled analgesia (PCA) or fixed interval i.v. administration of strong opioids should be preferred to on-demand administration according to the Procedure-Specific Postoperative Management (PROSPECT) recommendations ${ }^{4}$. However, the results of comparisons between PCA and conventional methods of analgesia have been inconsistent ${ }^{5,6}$. In patients undergoing THR, patient-controlled epidural analgesia (PCEA) may be considered an appropriate alternative for managing postoperative pain $\left(\right.$ ref. $\left.^{3}\right)$. However, other studies on postoperative pain management with PCEA used morphine despite the fact that sufentanil is a highly effective lipophilic opioid analgesic currently used more often in clinical practice ${ }^{7,8}$. Only a few studies have to date investigated the use of sufentanil for PCEA.

The primary goal of this prospective, randomized study was to compare the effects of two different sufentanil-based methods of analgesia; patient controlled (PCEA) and conventional non-PCEA) where the drug is delivered according to the physician's prescription. The main goal was to determine the difference in total use of analgesic mixture. The secondary goals were 1) patient satisfaction during the first $24 \mathrm{~h}$ postoperatively; 2) the degree of pain intensity; and 3) to assess the safety of the methods of analgesia based on the occurrence of analgesia-related complications including hypotension, bradypnea, heart rate abnormalities, itching, and postoperative nausea and vomiting (PONV). 
Table 1. Inclusion and exclusion criteria.

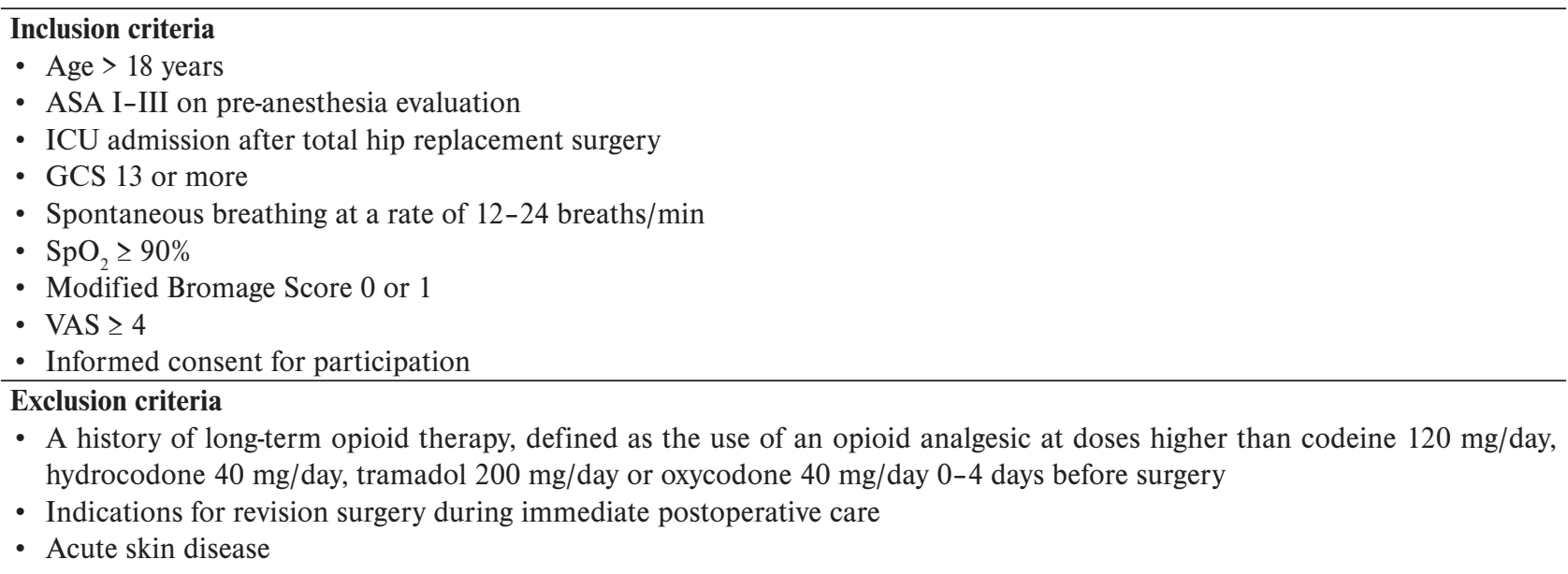

ASA, American Society of Anesthesiologists; GCS, Glasgow Coma Scale; ICU, intensive care unit; VAS, Visual Analogue Scale

\section{PATIENTS AND METHODS}

\section{Trial design and patients}

This prospective, randomized controlled trial was approved by the Ethics Committee of the University Hospital of Ostrava (Ref: 713/2013) and registered at clinicaltrial.gov (ID: NCT03599024). Patients were informed in person and written informed consent was then obtained from all participants prior to enrollment in the study in accordance with The Code of Ethics of the World Medical Association (Declaration of Helsinki) for experiments involving human subjects. The trial was conducted between September 2014 and March 2016 at the Department of Anesthesia and Intensive Care Medicine, University Hospital of Ostrava. All eligible participants were adult patients of both genders aged 18 years or older, and scheduled for elective orthopedic surgery (THR). A detailed list of inclusion and exclusion criteria is given in Table 1.

\section{Randomization procedure}

The enrolled patients underwent per-protocol randomization into one of two groups (PCEA or non-PCEA group) using the envelope method giving the participant a $50 \%$ chance of being assigned to either study or control group. Randomization was performed immediately after ICU admission by an independent physician who was not involved in the data collection or management of the study subjects.

\section{Variables and time intervals of measurement}

Following randomization, patients were admitted to the ICU. Data for the analyses were collected for $24 \mathrm{~h}$ after THR.

The baseline demographic and preoperative characteristics (age, gender, weight, body mass index, and American Society of Anesthesiologists [ASA] physical status) were obtained at the time of admission.

The degree of motor block was assessed using the modified Bromage Score ${ }^{9}$. The level of sedation due to the residual effect of anesthetics at the time of admis- sion was assessed using a sedation score ( 1 = awake; 2 = tired, sleepy, but easy to wake up; 3 = somnolent; and 4 = coma) (ref. ${ }^{10}$ ). The Visual Analogue Scale (VAS) values were determined at hourly intervals and $30 \mathrm{~min}$ after administration of analgesics to determine the effect of the analgesic dose. The other parameters evaluated at hourly intervals were: a) the presence of hypotension defined as a decrease in systolic blood pressure below $90 \mathrm{mmHg}$ or a greater than $30 \%$ decrease from the baseline value; b) bradypnea measured on a numeric scale: ( $1=$ normal respiratory rate $[\mathrm{RR}] ; 2=\mathrm{RR} \leq 12 / \mathrm{min}$; and $3=\mathrm{RR} \leq 8 /$ $\mathrm{min}$ ); c) bradycardia defined as heart rate $\leq 50 / \mathrm{min}$ and tachycardia defined as heart rate $\geq 120 / \mathrm{min}$; d) the presence of PONV as determined using a numerical scale $(0=$ no nausea; 1 = mild nausea; 2 = antiemetic given; $3=$ nausea despite antiemetic; and $4=$ vomiting) (ref. $\left.{ }^{11}\right)$; and e) skin itching (yes/no).

At the time of discharge from the ICU, the total use of analgesics was recorded, and patients' satisfaction was evaluated using Likert scale (5-point version) (ref. ${ }^{12}$ ).

\section{Protocol for administration of anesthesia and analgesia}

The patients were given $7.5 \mathrm{mg}$ of midazolam orally one hour before surgery. Patients weighing more than $70 \mathrm{~kg}$ received $2 \mathrm{mg}$ of bisulepin. Prior to surgery, subarachnoid blockade was established with 2-4 $\mathrm{mL}$ of levobupivacaine $0.5 \%$ at the L2-L3 spinal interspace. Subsequently, a catheter for postoperative analgesia was inserted into the epidural space. If the subarachnoid blockade was insufficient for surgery, epidural levobupivacaine $0.5 \%$ was provided to a maximum of $10 \mathrm{~mL}$, after which the patient underwent general anesthesia and was excluded from the study. During surgery, patients were sedated with a target-controlled infusion of propofol (dose of $1-2 \mathrm{mg} / \mathrm{kg}$ body weight per hour) so that they were asleep but aroused when spoken to. After surgery, patients received a mixture of levobupivacaine $0.1 \%$ and sufentanil $1 \mu \mathrm{g} / \mathrm{mL}$. Postoperatively, patients were moved to the ICU. Immediately after ICU admission, continuous monitoring of vital functions and pain was initiated. When the pain intensity exceeded $\geq 4$ points, analgesic 
Table 2. The baseline demographic and presurgical characteristics.

\begin{tabular}{|c|c|c|c|}
\hline Characteristics & $\begin{array}{l}\text { PCEA group } \\
\quad(n=55)\end{array}$ & $\begin{array}{l}\text { non-PCEA group } \\
\qquad(\mathrm{n}=56)\end{array}$ & $P$ \\
\hline Age, years, mean \pm SD & $65.5 \pm 9.4$ & $69.7 \pm 10.3$ & 0.032 \\
\hline Males \% (n) & $49.1(27)$ & $36(15)$ & 0.015 \\
\hline Females (n) \% & $50.9(28)$ & $64(41)$ & \\
\hline Body weight, $\mathrm{kg}$, mean $\pm \mathrm{SD}$ & $83.8 \pm 15.3$ & $78.3 \pm 14.8$ & 0.065 \\
\hline BMI, $\mathrm{kg} / \mathrm{m}^{2}$, mean $\pm \mathrm{SD}$ & $29.1 \pm 4.1$ & $28.4 \pm 5.2$ & 0.493 \\
\hline ASA I, \% (n) & $1.8(1)$ & $3.6(2)$ & 1.000 \\
\hline ASA II, \% (n) & $92.7(51)$ & $94.7(53)$ & 0.716 \\
\hline ASA III, \% (n) & $5.5(3)$ & $1.8(1)$ & 0.364 \\
\hline
\end{tabular}

Nominal values are reported as elative frequencies (in \%) and absolute numbers; numeric values are reported as means \pm standard deviation (SD). The $\mathrm{p}$ values indicate differences between the patient-controlled epidural analgesia (PCEA) group and the non-patient-controlled epidural analgesia (non-PCEA) group.

BMI, Body mass index; ASA, American Society of Anesthesiologist

therapy was initiated by administration of a mixture containing levobupivacaine $0.1 \%$ and sufentanil $1 \mu \mathrm{g} / \mathrm{mL}$. The PCEA group was initially given a bolus of $10 \mathrm{~mL}$ of the mixture then a basal infusion at a rate of $3 \mathrm{~mL} / \mathrm{h}$. A bolus was set on $4 \mathrm{~mL}$, a lockout interval of $20 \mathrm{~min}$, and a maximum dose of $40 \mathrm{~mL} / 4 \mathrm{~h}$ according to the literature recommendation ${ }^{13}$. The non-PCEA group was initially administered $5 \mathrm{~mL}$ of the analgesic mixture followed by a basal infusion at $5 \mathrm{~mL} / \mathrm{h}$. If pain developed, a bolus of $8 \mathrm{~mL}$ of the mixture was administered according to the physician's prescription. If analgesia was insufficient after one hour of maximal dosing in both groups, the patient

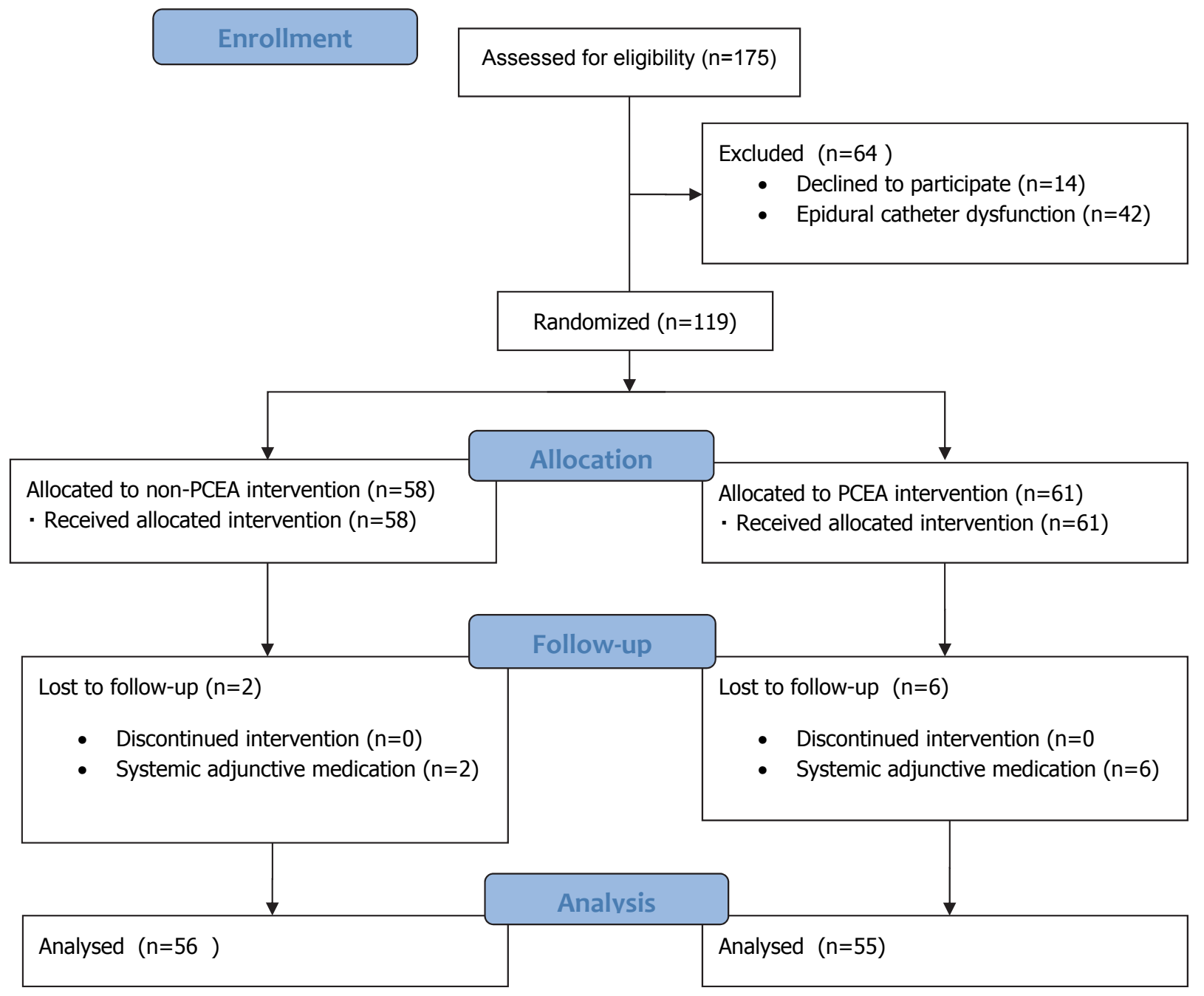

Fig. 1. CONSORT 2010 Flow Diagram. 


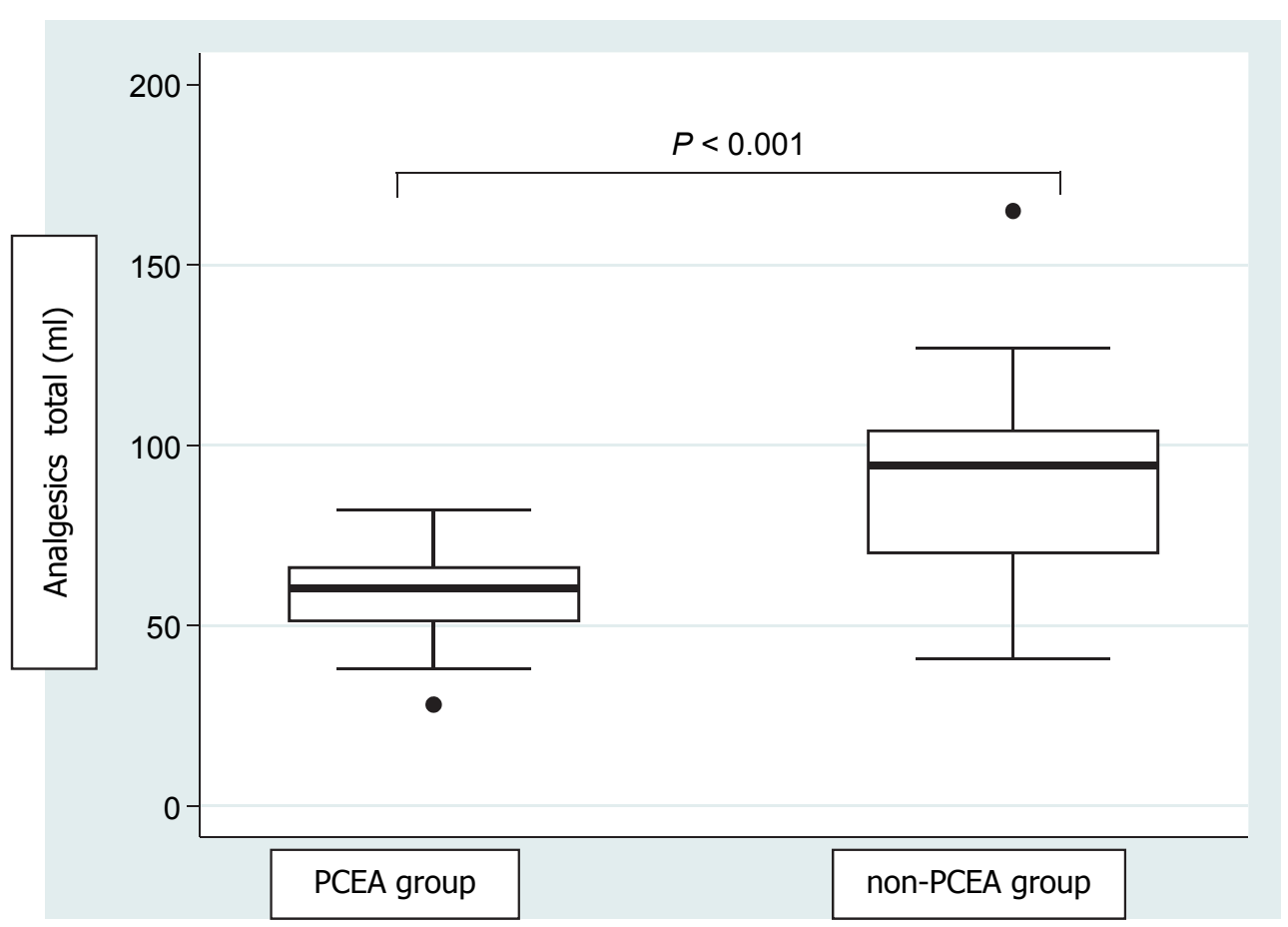

Fig. 2. Comparison of the total use of the analgesic mixture between the PCEA and non-PCEA group. PCEA, patient-controlled epidural analgesia; non-PCEA, (conventional) non-patient-controlled epidural analgesia.

was given adjunctive analgesic therapy including one or a combination of the following drugs: i.v. paracetamol, i.v. metamizole, or i.v. tramadol. Patients requiring systemic adjunctive medication were excluded from the final analysis.

\section{Statistical analysis}

A power analysis based on the authors' pilot data was performed prior to the study start. Continuous variables were expressed as means and standard deviation (SD) and categorical variabl es as proportions. Selected variables (anaesthetic mode) were compared using the nonparametric Mann-Whitney test and chi-square test or, where necessary, Fisher's exact test. Some data were graphically depicted as box plots. To address the baseline differences between groups, a regression analysis was performed including the influence of age, sex, and randomization. The level of significance was set at $5 \%$. The analyses were performed by a certified statistician using Stata 13 software.

\section{RESULTS}

During the study period, 175 patients undergoing THR were assessed for eligibility. The final analysis included data from 111 patients divided into the PCEA $(n=55)$ and non-PCEA $(n=56)$ groups. The CONSORT 2010 flow diagram of the patient selection process is shown in Fig. 1. The PCEA group was significantly younger $(P=0.032)$ and consisted of fewer males than females $(P=0.015)$. Other baseline and preoperative characteristics were not significantly different between the groups (Table 2).

\section{Total use of analgesics}

The total use of the analgesic mixture during the first $24 \mathrm{~h}$ after ICU admission was significantly lower in the PCEA group. The mean sum of the continuous and bolus doses was $0.9 \pm 0.3 \mathrm{~mL} / \mathrm{kg}$ in the PCEA group and $1.3 \pm 0.4 \mathrm{~mL} / \mathrm{kg}$ per day in the non-PCEA group $(P<0.001)$; i.e., $73.0 \pm 17.1 \mathrm{~mL}$ per day in the PCEA and $97.9 \pm 25.0 \mathrm{~mL}$ per day in the non-PCEA groups $(P<0.001$; Fig. 2$)$. A bolus of the analgesic mixture was administered to 50 patients $(91 \%)$ in the PCEA group and 46 patients $(82 \%)$ in the non-PCEA group $(P=0.18)$.

\section{Patient satisfaction during postoperative pain management}

The use of a Likert scale showed that the PCEA group was considerably more satisfied than the nonPCEA group, with mean satisfaction scores of $4.3 \pm 1.0$ and $2.8 \pm 0.7$, respectively $(P<0.001$; Fig. 3$)$. According to gender, the median satisfaction was higher in males $(3.9 \pm 1.2)$ than in females $(3.3 \pm 1.2), P=0.014$.

\section{Pain intensity}

The assessment of pain intensity using the VAS showed that mean VAS scores were similar in the PCEA and non-PCEA groups ( $1.1 \pm 0.6$ and $1.2 \pm 0.4$, respectively, $P=0.14$ ) during the first $24 \mathrm{~h}$ postoperatively. Mean VAS scores were less than 2 in the entire sample (both groups). We found that the PCEA group had lower intensity of pain for 16 hours of the following $24 \mathrm{~h}$ compared to $8 \mathrm{~h}$, where the VAS was non-significantly higher in the PCEA group. Significantly higher VAS scores in the non-PCEA group were found at only three time points: $6 \mathrm{~h}(P=0.02)$, 


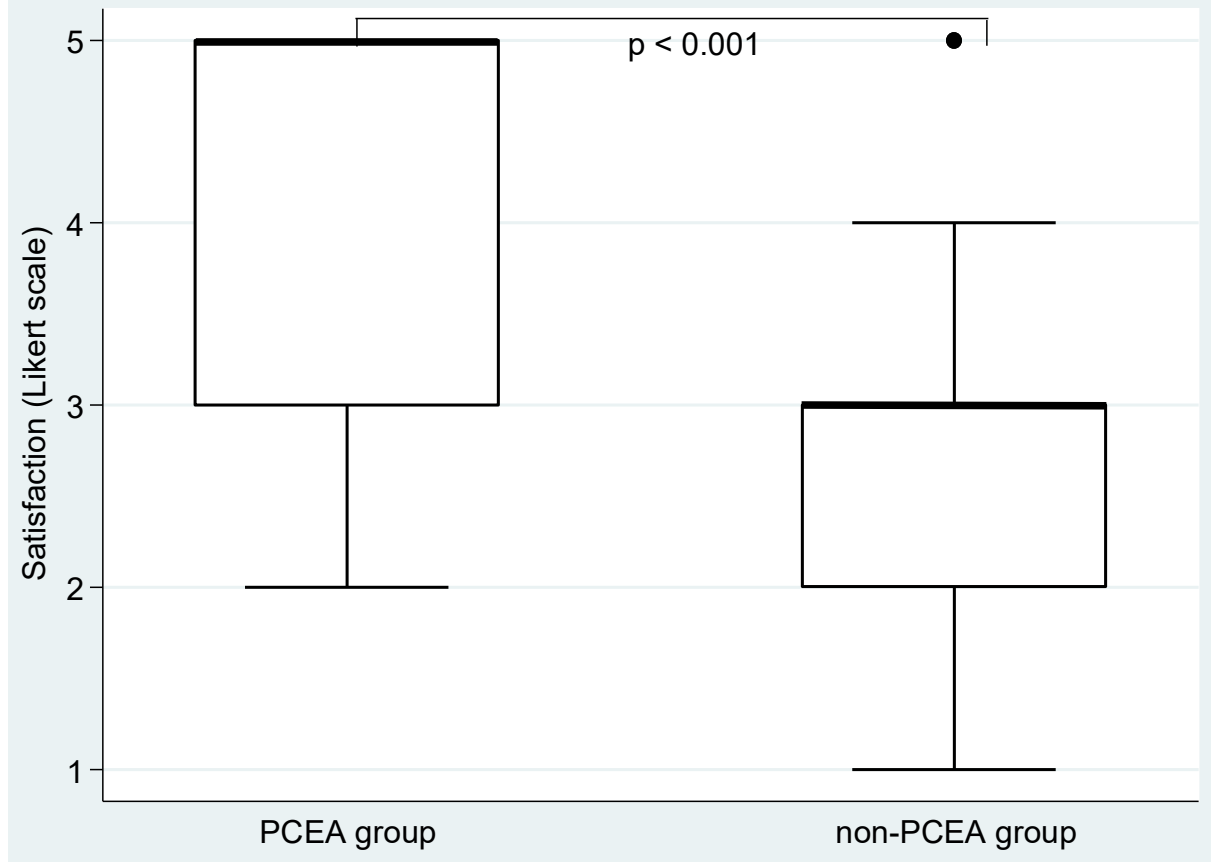

Fig. 3. Comparison of patient satisfaction between the PCEA and non-PCEA group. PCEA, patient-controlled epidural analgesia; non-PCEA, (conventional) non-patient-controlled epidural analgesia.

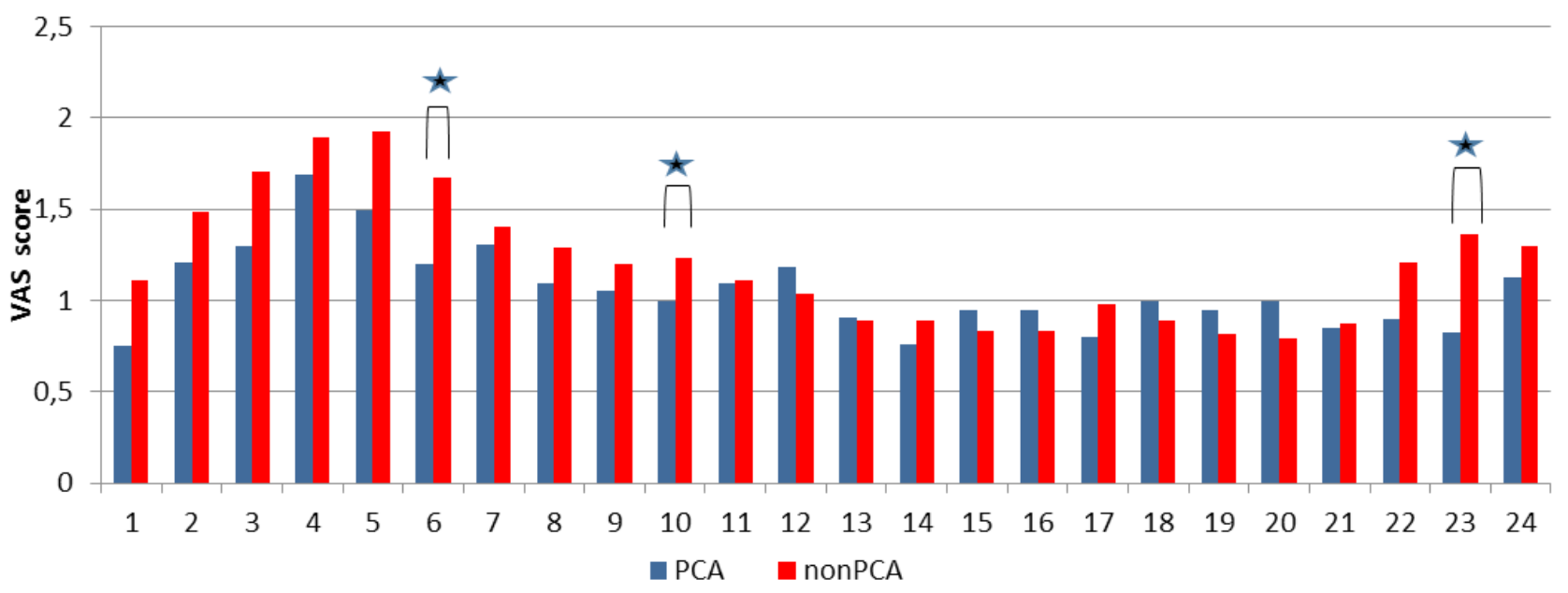

Fig. 4. Comparison of Visual Analogue Scale scores during first $24 \mathrm{~h}$ after surgery between the PCEA and non-PCEA group. PCEA, patient-controlled epidural analgesia; non-PCEA, (conventional) non-patient-controlled epidural analgesia; VAS, Visual Analogue Scale.

$10 \mathrm{~h}(P=0.01)$, and $23 \mathrm{~h}(P=0.03)$ after the initiation of analgesic therapy (Fig. 4).

\section{Analgesia-related complications}

Hypotension was noted in a total of 37 patients. The occurrence of hypotension in the PCEA group was $40 \%$ $(\mathrm{n}=22)$, while it was $26.8 \%(\mathrm{n}=15)$ in non-PCEA group $(P=0.14)$. Bolus-related hypotension was found in $20.0 \%$ $(n=10)$ of cases in the PCEA group and $8.7 \%(n=4)$ of cases in the non-PCEA group $P=0.15$ ). Bradypnea developed in $20.7 \%(n=23)$ of cases in the entire sample; $14.5 \%$ $(n=8)$ of cases in the PCEA group and $26.8 \%(n=15)$ of cases in the non-PCEA group $(P=0.11)$. No heart rate abnormalities (i.e. bradycardia and/or tachycardia), PONV, or any form of itching were observed in the whole group.

\section{DISCUSSION}

Individualized therapeutic management of patients is currently recommended in a majority of health care fields, and postoperative pain relief is an essential priority for all patients undergoing surgical procedures of any kind. In the field of hip arthroplasty the PROSPECT recommendations prefer PCA over analgesia on patient request ${ }^{4}$.The primary aim of our study was to compare the PCEA modality with standard pain management in patients undergoing THR. THR is a frequent and challenging orthopedic procedure usually followed by high levels of postoperative pain. We found lower total consumption of an analgesic mixture in the PCEA group during the first $24 \mathrm{~h}$ after THR. Moreover, higher subjective patient satisfaction 
was also found in the PCEA group. Both methods were effective in decreasing the pain intensity. There was no significantly different rate of analgesia-related complications between the two groups. Patients in the PCEA group tolerated the method well.

Seventy two percent of patients undergoing orthopedic surgery experience moderate to severe pain at rest, and $89.3 \%$ experience pain during early mobilization ${ }^{13}$. Untreated or poorly managed pain can delay the initiation of rehabilitation, ambulation, and basic self-care. Therefore, it significantly limits patients' return to normal daily routines. Patients who are in pain are also at risk of developing health complications due to prolonged bed rest such as thromboembolic diseases and pulmonary dysfunction ${ }^{14}$. Patients undergoing THR are usually treated by administration of strong opioids, sometimes in combination with non-opioid analgesics. The currently recommended and generally accepted modality for pain management in this group of patients is drug administration in the epidural space. It is associated with a better analgesic effect and a lower risk of complications compared to systemically administered analgesics ${ }^{15}$.

Concerning patients' active involvement in pain management, i.v. PCA was considerably more effective in reducing pain after cardiac surgery than nurse-controlled analgesia ${ }^{16}$. In acute traumatic pain in the emergency department, i.v. PCA with morphine was associated with faster pain relief and greater patient satisfaction than standard analgesia ${ }^{17}$. However, the current study was more concerned with a comparison among local anesthetics during PCA, and only a few studies have compared the effects of i.v. PCA (or PCEA) to the conventional mode of analgesia in patients undergoing THR. For instance, the effect of mixtures of sufentanil and ropivacaine $0.165 \%$ or levobupivacaine $0.125 \%$ was compared during PCEA after orthopedic surgery. Patients receiving sufentanil and levobupivacaine required lower doses of analgesics and were more satisfied due to greater pain relief ${ }^{18}$. Similar results were found in a randomized study comparing the effects of levobupivacaine $0.125 \%$, bupivacaine $0.125 \%$, and ropivacaine $0.2 \%$ administered via a PCEA mode $(5 \mathrm{~mL} / \mathrm{h}$ basal infusion, $2 \mathrm{~mL}$ bolus, $20 \mathrm{~min}$ lockout) in patients undergoing orthopedic surgery. With regard to pain intensity and optimal recovery of motor function, levobupivacaine appeared to be the most effective ${ }^{19}$. According to these results, the combination of levobupivacain and sufentanil was chosen for PCEA in our study.

The total consumption of analgesic is an important clinical end point because of the potential adverse effects of high doses of opioids ${ }^{20}$. Moreover, decreasing the dose of analgesics can also lead to a reduction in treatment costs and shorter time spent bedside by medical staff. We found that the PCEA modality of drug delivery significantly decreased the overall amount of analgesics necessary for required pain relief. To date, no study has compared the effect of PCEA on analgesic consumption after THR.

Postoperative pain management using i.v. PCA versus conventional methods of pain control was compared in a study that investigated patient satisfaction after surgical intervention. The duration of PCA pump use and patients' age, gender, marital status, educational level, type of surgery, and work status were identified as significant predictors of patient satisfaction $(P<0.001)$ (ref. $\left.{ }^{21}\right)$. Our findings also demonstrated higher satisfaction with the PCEA method of drug delivery. In another study, an association between satisfaction and a decrease in pain intensity was proposed ${ }^{22}$. However, satisfaction is subjective and objective measurement of it is controversial. For example, patient satisfaction surveys tend to yield positive results since patients are reluctant to criticize the treatment process, and even though many patients experience high levels of pain, they still report satisfaction with pain management $^{23}$. Assessment of satisfaction is often considered an important parameter of how patients perceive the efficacy of their treatment. However, in agreement with our results, a prospective study assessing patient satisfaction and outcome after general surgery concluded that PCEA was associated with higher satisfaction independent of the maximum pain intensity assessed by VAS score after orthopedic and gynecological surgery ${ }^{24}$. We presume that higher satisfaction ratings in our PCA group are related mostly to perceived possibility of higher control over pain relief given by PCA.

The results of studies addressing pain intensity are heterogeneous. The studies from 2001 concluded that the perception of pain by surgical patients who receive various types of analgesia is essentially identical. However, PCA helps patients feel more secure and less anxious ${ }^{25,26}$. In our study, the estimated pain intensity by VAS score showed generally lower VAS scores (16 of 24 postoperative hours) in the PCEA group versus the non-PCEA group during their 24-hour stay in the ICU. However, the overall VAS score did not exceed a value of 2 , and no statistical significance in mean VAS scores was found between the groups. These results suggest that pain management was successful using both modalities of analgesic administration. This highlights the importance of the decreased amount of analgesics used in the PCEA group, while the difference in pain intensity was insignificant between the groups.

The continuous administration of opioids requires constant monitoring of physiological functions. Opioid-based drugs cause more than $60 \%$ serious postoperative side effects, especially respiratory depression, which can lead to cardiac arrest and death $\left(\right.$ ref. $\left.^{20}\right)$. The use of PCEA offers easier pain management and decreases the time spent on patient care by qualified nursing staff ${ }^{27}$. To evaluate the incidence of analgesia-related complications, we monitored the occurrence of hypotension, bradypnea, heart rate abnormalities such as bradycardia and/or tachycardia, PONV, and postoperative itching. We expected that especially hypotension and bradypnea might follow the administration of a bolus dose of an analgesic. Although the bolus dose was more frequently administered in the PCEA group, we found no significant difference in the occurrence of hypotension and bradypnea between groups. This result shows that PCEA is as safe as the conventional mode of epidural analgesia. 
We are aware of several study limitations including the relatively small sample size and differences in baseline characteristics (age, gender). However, the differences between groups were assessed by multivariate regression analysis, providing an opportunity for randomization, age, and sex to influence the results. Only randomization had a significant influence on the variables of interest including total analgesic consumption and patient satisfaction. The PCEA group consisted of more males than the non-PCEA group. This could affect patients' overall satisfaction because males were significantly more satisfied with pain management than females.

\section{CONCLUSION}

The PCEA modality of delivery is associated with less total analgesic consumption, more satisfaction, and similar rates of adverse analgesia-related complications compared to the conventional mode of epidural analgesia after THR. Our results suggest that PCEA should be considered the method of first choice for analgesia in patients after THR.

Acknowledgement: The study was supported by local grant project: MH CZ - DRO-FNOs/2014.

Author contributions: All authors contributed to the data collection, analysis and writing of the manuscript. All authors have read and approved the final manuscript.

Conflict of interest statement: The authors state that there are no conflicts of interest regarding the publication of this article.

\section{REFERENCES}

1. Chen PP, Chui PT, Ma M, Gin T. A prospective survey of patients after cessation of patient-controlled analgesia. Anesth Analg 2001;92(1):224-7.

2. Sinatra RS, Torreas J, Bustos AM. Pain managements after major orthopedic surgery: current strategies and new concepts. J Am Acad Orthop Surg 2002;10(2):117-29.

3. Singelyn FJ, Ferrant T. Effects of intravenous patient-controlled analgesia with morphine, continuous epidural analgesia, and continuous femoral nerve sheath block on rehabilitation after unilateral total-hip arthroplasty. Reg Anest Pain Med 2005;30(5):452-57.

4. Procedure-Specific Postoperative Management (PROSPECT), Summary recommendations for total hip arthroplasty. https://www. postoppain.org/methodology/; 2017 [accessed 13 February 2018]

5. Ebneshahidi A, Akbari M, Heshmati B. Patient-controlled versus nurse-controlled post-operative analgesia after caesarean section. Adv Biomed Res 2012;1:6.

6. Hudcova J, Mc NE, Quah C, Lau J, Carr DB. Patient controlled opioid analgesia versus conventional opioid analgesia for postoperative pain. Cochrane Database Syst Rev 2006;18(4):CD003348.

7. Kissin I. Patient-Controlled-Analgesia Analgesimetry and Its Problems, Anesth Analg 2009;108(6):1945-49.

8. Hartrick CT, Bourne MH, Gargiulo K, Damaraju CV, Vallow S, Hewitt DJ. Fentanyl Iontophoretic Transdermal System for Acute-Pain Management After Orthopedic Surgery: A Comparative study With
Morphine Intravenous Patient-Controlled Analgesia, Reg Anest Pain Med 2006;31(6):546-55

9. Bertini L, Mancini S, Di Benedetto P, Ciaschi A, Martini O, Nava S, Tagariello V. Postoperative Analgesia by Combined Infusion and Patient-Controlled Epidural Analgesia (PCEA) following Hip Replacement: Ropivacaine versus Bupivacaine, Acta Anaesthesiol Scand 2001;45(6):782-85.

10. Schnabel A, Poepping DM, Gerss J. Sex-related Differences of PatientControlled Analgesia for Postoperative Pain, Pain 2012;153(1):23844.

11. Ebell MH. Point-of-Care Guides, Predicting Postoperative Nausea and Vomiting. Am Fam Physician 2007;15(10):1537-38.

12. Pennington $P$, Caminiti S, Schein JR, Hewitt DJ, Nelson WW. Patients' Assessment of the Convenience of Fentanyl $\mathrm{HCl}$ Intophoretic Transdermal System (ITS) Versus Morphine Intravenous PatientControlled Analgesia (IV PCA) in Management of Postoperative Pain After Major Surgery, Pain Manag Nurs 2009;10(3):124-33.

13. Massad IM, Mahafza TM, Abu-Halawah SA, Attyyat BA, AIGhanem SM, Almostafa MM, Sal-Oweidi AS. Postoperative Pain is Undertreated: Results from a Local Survey At Jordan University Hospital, East Mediterr Health J 2013;19(5):485-99.

14. Rokyta R, Kršiak M, Kozák J. Pain - revised additive edition, Praha: TIGRIS; 2012. ISBN 978 80-87323-02-1.

15. Block BM, Spencer SL, Rowlingson AJ. Efficacy of Postoperative Epidural Analgesia a Meta-analysis. JAMA 2003;290(18):2455-63.

16. Pettersson PH, Lindskog EA, Owall A. Patient-Controlled versus Nurse-Controlled Pain Treatment after Coronary Artery Bypass Surgery. Acta Anaesthesiol Scand 2000;44(1):43-7.

17. Rahman DH, De Silva T. A Randomized Controlled Trial of PatientControlled Analgesia Compared with Boluses of Analgesia for The Control of Acute Traumatic Pain in the Emergency Department, Acta Anaesthesiol Taiwan 2012;50(3):92-5.

18. Smet I, Vlaminck E, Vercauteren M. Randomized Controlled Trial of Patient-Controlled Epidural Analgesia After Orthopaedic Surgery with Sufentanil and Ropivacaine $0.165 \%$ or Levobupivacaine 0.125\%. Br J Anaesth 2008;100(1):99-103.

19. Casati A, Santorsola R, Aldegheri G, Ravasi F, Fanelli G, Berti M, Fraschini G, Torri G. Intraoperative Epidural Anesthesia and Postoperative Analgesia With Levobupivacaine for Major Orthopedic Surgery: A Double-blind, Randomized Comparison of Racemic Bupivacaine and Ropivacaine, J Clin Anesth 2003;15(2):126-31.

20. Benyamin R, Trescot AM, Datta S, Buenaventura R, Adlaka R, Sehgal N, Glaser SE, Vallejo R. Opioid Complications and Side Effects. Pain Physician. 2008;11(2 Suppl):S105-20.

21. Hamza FR, Ayman M, Samer HMS, Seder W, Salim M. Patient Satisfaction About Using Patient Controlled Analgesia in Managing Pain Post Surgical Intervention, Clin Nurs Res 2014;23(4):353-68.

22. McNicol ED, Ferguson M, C, Hudcova J. Patient controlled opioid analgesia versus non-patient controlled opioid analgesia for postoperative pain. Cochrane Database Syst Rev 2015;(6):CD003348.

23. Jamison RN, Taft K, O'Hara JP, Ferrante FM. Psychosocial and Pharmacologic Predictors of Satisfaction with Intravenous PatientControlled Analgesia. Anesth Analg 1993;77(1):121-5.

24. Gottschalk A, Freitag M, Liehr K, Domke A, Schuster M, Standl T. Does patient satisfaction correlate with pain level during PatientMonitored Epidural Analgesia. Evaluation of data from postoperative pain service. Schmerz 2004;18(2):145-50.

25. Mclntyre PE. Safety and Efficacy of Patient-Controlled Analgesia. J Pain Symptom Manage 2001;87(1):36-46.

26. Walder B, Schafer M, Henzi I, Tramer MR. Efficacy and Safety of Patient-Controlled Opoid Analgesia for Acute Postoperative Pain: A Quantitative Systematic Review. Acta Anaesthesiol Scand 2001;45:795-804.

27. King S, Walsh K. I think PCA is great, but... '- Surgical Nurses' Perceptions of Patient Controlled Analgesia. Int J Nurs Pract 2007; 13:276-83. 